\title{
Designing a Chatbot for Survivors of Sexual Violence: Exploratory Study for Hybrid Approach Combining Rule-based Chatbot and ML-based Chatbot
}

\author{
Wookjae Maeng \\ Seoul National University \\ Republic of Korea(South Korea) \\ ideaplayer@snu.ac.kr
}

\author{
Joonhwan Lee \\ Seoul National University \\ Republic of Korea(South Korea) \\ joonhwan@snu.ac.kr
}

\begin{abstract}
Chatbots are developed using two approaches: rule-based and MLbased. The former has a scripted tree structure consisting of questions and answers, while the latter can understand the intent of a user's question and generate answers from machine learning. Despite these benefits, each method has drawbacks: rule-based chatbots cannot answer slightly different questions with the same meaning in the patterns, and ML-based chatbots have difficulty performing continuous conversations while keeping the conversation context. We propose the concept of a hybrid chatbot that combines a rule-based chatbot and an ML-based chatbot to overcome the limitations of each. We conducted an exploratory study to investigate how a hybrid chatbot could support a survivor of sexual violence. A total of 349 questions asked from the stance of survivors were collected and analyzed. We found that the most frequently asked questions were about the punishment for sexual violence, the police report, and the list of support centers. Also, $30 \%$ of the questions omitted contextual information about sexual violence, and $10 \%$ of questions consisted of only keywords, not complete sentences. We suggest two design implications for the hybrid chatbot based on the survey's findings: 1) dialog flow for helping survivors understand the abuse experience sufficiently to report the sexual violence to police, and 2) contextualization strategies for connecting dialog flow with questions from the survivors.
\end{abstract}

\section{CCS CONCEPTS}

- Human-centered computing $\rightarrow$ Empirical studies in $\mathrm{HCI}$; Ubiquitous and mobile computing systems and tools.

\section{KEYWORDS}

chatbot, hybrid chatbot, sexual violence, survivors of sexual violence

ACM Reference Format:

Wookjae Maeng and Joonhwan Lee. 2021. Designing a Chatbot for Survivors of Sexual Violence: Exploratory Study for Hybrid Approach Combining

${ }^{*}$ Corresponding author

Permission to make digital or hard copies of all or part of this work for personal or classroom use is granted without fee provided that copies are not made or distributed for profit or commercial advantage and that copies bear this notice and the full citation on the first page. Copyrights for components of this work owned by others than ACM must be honored. Abstracting with credit is permitted. To copy otherwise, or republish, to post on servers or to redistribute to lists, requires prior specific permission and/or a fee. Request permissions from permissions@acm.org.

Asian CHI Symposium 2021, May 8-13, 2021, Yokohama, Japan

(c) 2021 Association for Computing Machinery.

ACM ISBN 978-1-4503-8203-8/21/05 . \$ \$15.00

https://doi.org/10.1145/3429360.3468203
Rule-based Chatbot and ML-based Chatbot. In Asian CHI Symposium 2021 (Asian CHI Symposium 2021 ), May 8-13, 2021, Yokohama, Japan. ACM, New York, NY, USA, 7 pages. https://doi.org/10.1145/3429360.3468203

\section{INTRODUCTION}

Recently, chatbots have become so popular in various fields such as customer services [8], psychological counseling [12], and surveys [17]. The prevalence of chatbots is based on advancements in natural language processing (NLP) and chatbot builders. NLP enables chatbots to understand human language better, and chatbot builders enable people to develop chatbots quickly.

There are two approaches in developing chatbots: rule-based and machine learning(ML)-based. A rule-based chatbot has a scripted tree structure consisting of questions and answers. The rule-based chatbot anticipates specific answers to their specific questions, thereby leading the conversation with users. Although the rulebased chatbot has a less flexible conversation flow, this chatbot is suitable for performing specific tasks. However, rule-based chatbots cannot answer slightly different questions with the same meaning in the patterns. On the other hand, ML-based chatbots are more flexible to answer a variety of users' questions. An ML-based chatbot can grasp a question's intent before deriving the response. Despite understanding the users, users have difficulty performing continuous conversations with an ML-based chatbot because ML-based has trouble keeping the conversation context.

We propose a hybrid chatbot that combines a rule-based chatbot and an ML-based chatbot to reduce chatbots' limitations. In other words, the hybrid chatbot can overcome the limitation of rule-based chatbots by handling the users' questions outside the patterns and complement the drawback of ML-based chatbots by performing continuous dialogue flows.

We conducted an exploratory study to present design implications for hybrid chatbots for supporting a survivor of sexual violence. Many survivors of sexual violence remained silent about their experiences [16]. "Silence is caused by mistrust, not by choice" [11], and this silence was related to the low reporting rates. Although some survivors of sexual abuse wanted to talk about their experience, they were reluctant to have a face-to-face conversation [11]. Non-anonymity was one of the reasons why the survivors found it difficult to share their experiences in person. Conversation with chatbots is less a burden than a face-to-face conversation due to anonymity [15]. The survivors of sexual abuse can disclose to the chatbot the stories that they would not tell anyone.

This research conducted a survey to collect questions asked from the stance of survivors of sexual violence. We gathered 349 questions from 14 participants who asked questions to acquire 
information regarding survivors' situations. Thirteen categories emerged from the survey, and two distinctive features of the questions were found. We suggest design implications for hybrid chatbots based on the findings from the survey.

The rest of this paper reviews related work on the history of the chatbot, sexual violence, and chatbots for supporting survivors of sexual abuse. This paper describes how three versions of chatbot can support survivors of sexual violence: rule-based chatbot, MLbased chatbot, and hybrid chatbot. Then, the study design and results of the survey are reported. At last, design implications based on the result are presented.

\section{RELATED WORK}

\subsection{The history of chatbot}

The first chatbot ELIZA [29], developed in 1966, served as a psychotherapist who asked follow-up questions by analyzing the keywords from users' input. Although ELIZA had difficulty asking pertinent questions to all users' answers, it showed a better ability to communicate with users than expected with simple rules such as pattern matching. In 1988, Jabberwacky [25] had the self-learning ability to have conversations with users instead of following predefined rules. This chatbot found the most likely sentence used in similar contexts by searching all previous conversations. In 1995, ALICE [27] called "the most human computer," showed significant improvement over previous chatbots. Artificial Intelligence Markup Language was used to build ALICE, and this markup language impacted the descendant. There were more than 50,000 rules that were manually hard-coded in the public domain ALICE. Defining new rules to cover various users' input was a time-consuming and limited adaptation to new domains [27]. Retrieval-based modules were incorporated into rule-based modules to overcome the limitations of scaling up a rule-based chatbot. SmarterChild [20, 30] was the first popularized entertaining chatbot built-in Windows messenger in 2001, approximately 30 million people received stock information and sports events retrieved from the database. In 2010, a virtual assistant Siri, made by Apple, answered various questions from users' voice commands. Since then, major IT companies have launched chatbots such as Microsoft Cortana, Amazon Alexa, Google Assistant, and IBM Watson.

In NLP, language models, powered by machine learning, played a crucial role in the evolution of virtual assistants. Bidirectional encoder representations from transformers(BERT) [9], a deep learning language model, showed significant improvement over previous language models in almost all aspects. BERT even achieved higher scores than humans in the reading comprehension test. Although ML-based chatbots could grasp a question's intent before deriving the response, these chatbots had difficulty performing continuous conversations. ML-based chatbots had trouble keeping the conversation context because their responses were not sequentially connected.

Rule-based chatbots could be integrated into ML-based chatbots to overcome the limitation of short conversations because rule-based chatbots could have continuous conversations while keeping the context of the conversation by asking and answering in pre-scripted order. Although the rule-based chatbot has a less flexible conversation flow, this chatbot is suitable for conducting predetermined tasks.
Therefore, a hybrid chatbot that combines an ML-based chatbot with a rule-based chatbot is an appropriate design approach to develop chatbots for understanding the human language better and conducting predetermined tasks.

\subsection{Survivors of Sexual violence}

Sexual violence occurs all over the world, regardless of gender equality [1]. $94 \%$ of survivors of sexual violence were reported to have suffered from post-traumatic stress disorder(PTSD) [26], and $33 \%$ of victims of sexual assault were found to attempt suicide [19].

Even if the prevalence of sexual violence, the stigma against disclosures of sexual violence often prevented the survivors from disclosing their cases to anyone [14]. Instead of accessing facilities, some survivors sought support and advice from anonymous online communities such as Reddit [2, 7]. It was found that some survivors 'threw away' their accounts not to be identified by others after obtaining information [3].

Recently, many women stood in solidarity with others by posting their experience of sexual violence with the hashtag \#MeToo on Safecity, and social media [18]. In addition to \#MeToo, \#WhyIDidntReport movement shared the reasons why the survivors had not reported. Based on the literature review, there were eight reasons why the survivors did not report to the police: lack of information, shame, denial or minimization, fear of consequences, hopelessness or helplessness, drug/disassociation, protecting assailants, and young age [13]. Many survivors of sexual violence did not know how to report, where to report and to whom they could report their cases [28]. Besides, potential reporters mentioned that 'lack of evidence' was the main reason for not reporting [5]. Therefore, it was essential to inform the survivors of how to report their cases and gather evidence after the incident.

\subsection{Chatbot for assisting survivors of sexual violence}

Conversational agents were reported that they could be preferable conversation partners to talk about sensitive topics than humans. People felt more comfortable discussing a sensitive topic with a conversational agent because the conversational agent could not judge them [22]. Also, keeping the privacy and anonymity of responses increased individuals' willingness to answer sensitive questions [15].

There have been developing chatbots for survivors of sexual violence after \#MeToo movement. \#MeTooMaastricht [4] extracted the information, such as date and place, from postings on social media to help the survivors of sexual harassment. Although this chatbot extracted essential facts about the harassment, the chatbot assumed that the survivors had posted their experience in detail on social media. Spot [21] tracked sexual harassment in the workplace and organized their experience of harassment into a formallooking report. Lawbot [24] helped victims of sexual offenses by providing options to handle the situation. Although chatbots helped the survivors of sexual violence, little work was done to address the problem with the hybrid approach. The hybrid chatbot can understand the survivors' intentions and connect their intention to relevant conversation flows, thereby supporting them.

We reviewed the literature on the history of the chatbot, sexual violence, and chatbots for the survivor of sexual violence. This study 
(a) Rule-based chatbot

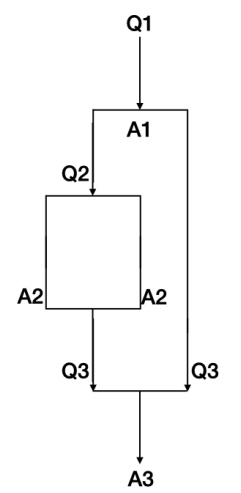

(b) ML-based chatbot

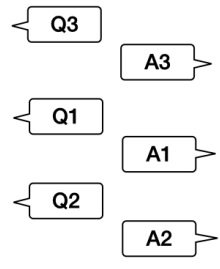

(c) Hybrid chatbot

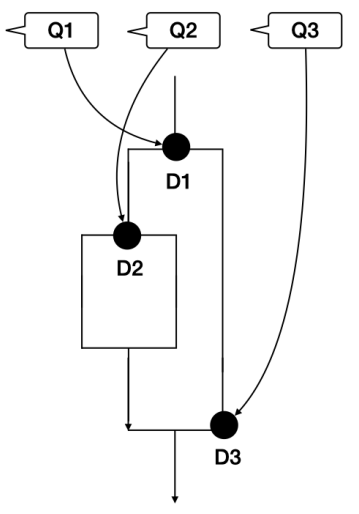

Figure 1: Three version of chatbots (a) rule-based chatbot, (b) ML-based chatbot, (c) hybrid chatbot

aims to help the survivors of sexual violence take action against offenders instead of remaining silent. In this regard, a chatbot can help the survivors have conversations on acquiring information while keeping their anonymity. To achieve the study's goal, we conducted an exploratory study to discover how a hybrid chatbot could support the survivors of sexual abuse. As the first step, we focused on design implications for the hybrid chatbot supporting the survivors of sexual violence.

\section{THREE VERSIONS OF THE CHATBOT FOR SUPPORTING SURVIVORS OF SEXUAL VIOLENCE}

A rule-based chatbot is developed to follow a scripted tree structure. As shown in (a) in Figure 1, if a rule-based chatbot asks the first question(Q1) to users, the users answer(A1) to the question by selecting predefined options. As the result of A1, the rule-based chatbot can ask follow-up questions such as Q2 and Q3. This chatbot is called a task-oriented chatbot because a rule-based chatbot is suitable for conducting predetermined tasks. Also, rule-based chatbots can maintain the context of the conversation by asking and answering in pre-scripted order. A typical example of a taskoriented chatbot is a pizza ordering bot. It is helpful for survivors of sexual violence to follow the dialog when they have no idea how to start conversations. However, the survivors can fail to find solutions to their problems when there is no pre-scripted conversation. Furthermore, rule-based chatbots repeat fallback messages such as 'I can't understand what you mean' when the survivors ask slightly different questions with identical meanings in prepared rules.

ML-based chatbots can understand the intent of users through technologies such as deep learning. Different from the rule-based chatbot, ML-based chatbots can answer slightly different questions with identical meanings. As shown in (b) in Figure 1, if a user asks a question(Q3) to an ML-based chatbot, this chatbot can answer(A3) to the questions. Users can ask questions in any order because there is no predefined order in the conversation between users and an ML-based chatbot. This chatbot is called chat-oriented because it is suitable for having a chit-chat with users. Survivors of sexual abuse can obtain information on their incident by asking various questions to ML-based chatbots. However, the survivors may not get answers because ML-based chatbots fail to understand the survivors' intentions. Moreover, the survivors can receive answers which are irrelevant to their questions when the chatbot has not learned relevant answers. Additionally, ML-based chatbots have difficulty performing continuous dialogue flows such as sorting out the survivors' experience to report to the police.

Hybrid chatbots combine the merits of rule-based chatbots and ML-based chatbots. As shown in (c) in Figure 1, if a user asks a question(Q1), a hybrid chatbot continues the conversation by connecting the question and related dialog flow(D1). Because MLbased modules in a hybrid chatbot can answer various questions having the same meanings, the hybrid chatbot can complement the rule-based chatbot that cannot handle the questions outside the patterns. The hybrid chatbot can also complement the ML-based chatbot as the former can continue the conversation when the survivors ask questions regarding their experiences. For example, the hybrid chatbot can grasp survivors' intention and guide the contextualized dialogue flows(e.g., gathering evidence of imagebased sexual violence in response to a question like "How can I report revenge porn?").

Therefore, the hybrid chatbot is the most suitable for helping the survivors of sexual assault among the three versions of chatbots.

\section{METHOD}

To derive design implications for the hybrid chatbot, we designed a survey to collect questions from the stance of survivors of sexual violence.

\subsection{Ethical Consideration}

The research design protocol was reviewed and approved by the institutional review board of our university, and we strictly followed the protocol to conduct user study. We recruited participants by posting an announcement on our institution's online community website and social media. Because of COVID 19 pandemic, we proceeded the whole process of user study online to safely protect 
Table 1: Nine questionnaires of the survey for sexual violence cases

\begin{tabular}{|c|c|c|}
\hline type & situation & participants \\
\hline \multirow{3}{*}{1} & discovering a hidden cam & \multirow{3}{*}{$\mathrm{P} 1, \mathrm{P} 10$} \\
\hline & deepfake & \\
\hline & reporting to the police for the first time & \\
\hline \multirow{3}{*}{2} & unlawful filming & \multirow{3}{*}{$\mathrm{P} 2, \mathrm{P} 11$} \\
\hline & forced disclosure of personal information online & \\
\hline & legal terms & \\
\hline \multirow{3}{*}{3} & sexually offensive comments on social media & \multirow{3}{*}{ P3,P12 } \\
\hline & sexual assault from a family member & \\
\hline & checking punishment & \\
\hline \multirow{3}{*}{4} & sharing intimate images/videos & \multirow{3}{*}{$\mathrm{P} 4, \mathrm{P} 13$} \\
\hline & cyberstalking & \\
\hline & support centers & \\
\hline \multirow{3}{*}{5} & re-sharing intimate images/videos & \multirow{3}{*}{$\mathrm{P} 5, \mathrm{P} 14$} \\
\hline & obscene text messages & \\
\hline & long-ago sexual violence & \\
\hline \multirow{3}{*}{6} & sharing intimate images/videos without consent & \multirow{3}{*}{ P6,P15 } \\
\hline & impostor account & \\
\hline & sexual violence under the age of 18 & \\
\hline \multirow{3}{*}{7} & blackmail for sharing intimate images/videos & \multirow{3}{*}{$\mathrm{P} 7, \mathrm{P} 16$} \\
\hline & sexual abuse by strangers in messenger & \\
\hline & anonymous counseling & \\
\hline \multirow{3}{*}{8} & sexual harassment in online games & \multirow{3}{*}{ P8,P17 } \\
\hline & sending sexual pictures on messenger & \\
\hline & removing intimate images/videos on websites & \\
\hline \multirow{3}{*}{9} & unlawful filming by acquaintances & \multirow{3}{*}{$\mathrm{P} 9, \mathrm{P} 18$} \\
\hline & making a statement & \\
\hline & gathering evidence for reporting to the police & \\
\hline
\end{tabular}

participants. Any participants could access the online research consent form, including the protocol, and voluntarily consent to the form. In the research consent form, we informed that participants could recall past unpleasant experiences by reading the scenario in the survey. If the participants experienced any discomforts during the survey, we asked them to withdraw the survey immediately and contact us to guide them to counseling centers in our university. If a participant suffered from psychological damages in the study, we would take psychological treatment procedures in medical centers, and researchers would handle the cost. We also informed that participants' data would be secured, and only the researcher could access their data. We did not collect identifiable information of our participants, such as name and affiliation. We removed any personal information in the responses.

\subsection{Participant}

Participants who responded were 14 females, ranging from the 20 s to $40 \mathrm{~s}$. Nine of them were graduate students, and five of them were employees.

\subsection{Survey}

To design a scenario-based survey presenting the verisimilar situation of sexual violence to the participants, we referred to the chatbot made by project ReSET [23], an organization fighting against blackmail and cybersex trafficking in messengers Telegram. We created 27 fictional cases of sexual violence from the Q\&A list in the chatbot and grouped those cases into nine different questionnaires for presenting various cases of sexual violence to participants (Table 1). Nine questionnaires were sent to nine groups, each consisting of two participants, via a Google survey form. Every participant was asked to fill out ten questions per sexual violence case, and each of them created 30 questions in total. We designed a between-subject study to protect all participants from being exposed to all cases of sexual violence in the questionnaires.

We created the scenario-based survey to help participants develop empathy for survivors based on facts and emotion, referring to the previous study on survivors of sexual violence [10]. For example, in the survey on finding a hidden camera, the incident presented as follows: 'You found the hidden camera in a public restroom.' The incident's emotions were presented as follows; 'You were so panicked that you did not know how to handle the hidden camera.' Lastly, the survey requests participants to write questions such as 'What will you ask chatbot as the survivors of sexual violence?'

\section{RESULTS}

We collected 420 questions from the stance of survivors of sexual violence from 14 participants. 71 questions out of 420 were invalid because they were duplicated or empty. We analyzed valid 349 
Table 2: The number of questions and their ratio based on the open-coded result

\begin{tabular}{lll}
\hline category & the number of questions & ratio(\%) \\
\hline punishment & 84 & 24 \\
\hline reporting & 59 & 17 \\
\hline support centers & 34 & 10 \\
\hline gathering evidence & 31 & 9 \\
\hline coping methods & 28 & 8 \\
\hline removing materials & 26 & 7 \\
\hline confirming the extent of harm & 25 & 7 \\
\hline emotional expression & 17 & 5 \\
\hline next steps & 17 & 5 \\
\hline similar cases & 12 & 3 \\
\hline law terms & 11 & 3 \\
\hline describing damage & 2 & 1 \\
\hline related information & 2 & 1 \\
\hline
\end{tabular}

questions based on grounded theory [6]. At first, we tagged 349 questions with codes that concisely summarized and represented the entire questions. After open coding, we created 96 codes and reviewed the codes grouped into higher-level themes. By combining the relevant tags, we yielded 13 categories from the data.

\subsection{Contents analysis}

As a result of the open coding based on grounded theory, 349 questions asked from the stance of survivors of sexual violence were divided into 13 categories (Table 2). The highest number of questions(84) was 'punishment,' occupying $24 \%$ of all questions. These are followed by 'reporting'(59), 'support centers'(34), 'gathering evidence'(31), 'coping method'(28), 'removing materials'(26), 'confirming the extent of harm'(25), 'emotional expression'(17), 'next steps'(17), 'similar cases'(12), 'law terms'(11), 'describing damage'(2), and 'related information'(2).

Specifically, questions on 'punishment' were related to clauses, compensation, and statute of limitations. In the 'reporting' category, questions were related to method, anonymity, contact point, timing, and the fee for reporting. The 'support center' category consists of psychological counseling, a list of the nearby support centers, anonymous counseling, reporting support, and counseling fee. Questions on 'gathering evidence' were related to gathering evidence first and how to cope with having no evidence. In the case of 'removing the materials', there were ways to quickly delete the shared images, monitor them, delete those materials permanently, and identify the victims in the images/videos. 'Confirming the extent of harm' consists of the offenders' arrest and the estimation of harm. In the category of 'emotional expression,' most questions related to the anxiety of revenge and light punishment concern. 'Similar cases' consist of questions on relevant cases from A to Z 'Law terms' was about complicated law terminologies. 'Describing damage' was not a question but a description of their damage, such as "My personal information seems to have been leaked." 'Related information' was the requests for information on their cases, such as "Give me information on stalking."

Participants asked questions without explaining the context of the harm, such as the type of sexual crime. For example, participants asked the chatbot the following: "Can you tell me about a case similar to mine?" or "How can I get him to be punished?". We found that 103 questions (30\%) were omitting the contextual information. Some participants asked questions to the chatbot by entering keywords instead of complete sentences. Thirsty-five (10\%) questions consisted of only keywords.

\section{DESIGN IMPLICATIONS FOR HYBRID CHATBOT}

\subsection{Designing conversation flows helping the process of reporting}

As a result of the survey, it turned out that the questions asked from the stance of survivors of sexual violence concerned 'punishment,' 'reporting,' 'support centers', 'gathering evidence,' and so on. This result echoed the findings in the previous work that survivors of sexual violence were unaware of how to report, where to report, and to whom they could report their cases [28]. Thus, we should help the survivors not to remain silent on their sufferings and to overcome their unawareness about reporting. As 'punishment,' 'reporting,' 'support centers' and 'gathering evidence' occupy over half of all questions, we should focus on designing hybrid chatbots for 'assisting the process of reporting for punishment.' Significantly, the chatbot's non-judgemental characteristic can help the survivors disclose sexually abused experiences [22]. Also, 'lack of evidence' was survivors' main reason for not reporting [5]. Therefore, we suggest the conversation flow starts from collecting evidence to reporting for punishment [5]. A visual diagram to show the main phases of the conversation flow for the hybrid chatbot is shown in Figure 2.

Because collecting evidence of sexual violence depended on their incident, conversation flow should be designed to make the survivors select the type of their sexual violence. The hybrid chatbot could infer the type of sexual abuse from the questions the survivors might ask. For example, the chatbot could recognize the type of sexual crime as stalking when the survivors asked, such as "What is the minimum sentence of stalking in California?" After recognizing the type, the chatbot explained how to gather proof of their sexual violence in a dialog flow. The survivor could learn 


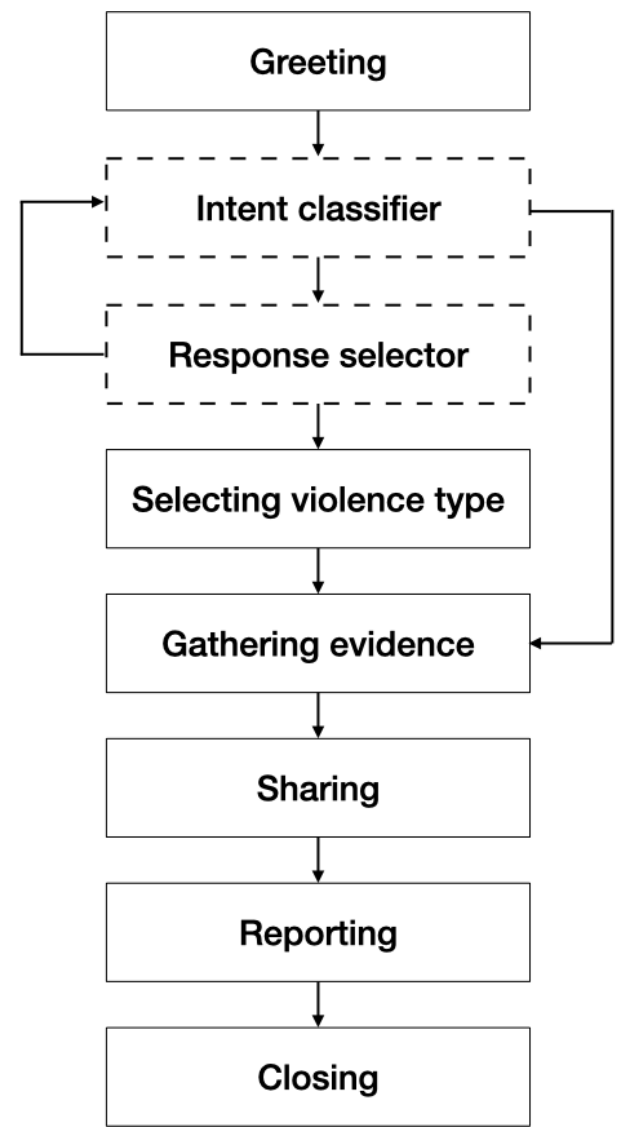

Figure 2: Main phases of hybrid chatbots conversation flow

how to gather testimony from the conversation and could apply the knowledge to collect the evidence of their incident directly. Then, the chatbot led the survivors to sort out their experiences by following a scripted conversation. The scripted conversation consisted of questions on the date and place of their incident and requested a detailed description of the experience. The survivors could organize the facts about their harm and prepare the reporting process while conversing with the chatbot. The investigation could properly proceed because the survivors reported on the crime.

\subsection{Specifying contextual information of questions}

Of all questions in the survey, $30 \%$ did not include the contextual information on sexual violence. This result implied that survivors of sexual violence could ask questions to a chatbot that lack specific contextual information of harm. Because the survivors could have difficulty describing their situation due to PTSD [26]. It was difficult even for humans to precisely answer the questions without the contextual information. A hybrid chatbot should fill in the question's contextual information to better connect the survivors' questions to conversation flow. We proposed two ways of filling in the contextual information in the question by the chatbot. First, a hybrid chatbot should be designed to specify the contextual information by reversely asking survivors about the context of their situation. For example, when the survivor asks a question such as 'Can I see a similar case with mine?', the chatbot re-asks the question 'What kind of cases do you want to know?' to determine the contextual information. Second, a hybrid chatbot should be designed to apply the contextual information in previous questions to the next question. Specifically, when the survivor asks questions in a row such as 'How can I report the revenge porn to the police?' and 'What kind of evidence do I need to collect?", the hybrid chatbot should guide the survivor to gather the evidence in the context of image-based sexual violence.

\section{FUTURE WORK}

A hybrid chatbot supporting survivors of sexual violence will be developed based on the design implications of this work. Moreover, the chatbot's effectiveness will be evaluated in terms of helping survivors report to the police.

Because this chatbot covers sexual violence, how to build trust between the survivors and chatbots and how to prevent the conversation from exposing to others should be considered in future work.

\section{CONCLUSION}

We proposed the concept of a hybrid chatbot and derived the design implication of that chatbot. To this end, we collected and analyzed questions asked from the stance of survivors of sexual violence. Survey results indicated that 'punishment,' 'reporting,' 'support centers' and 'gathering evidence' were the most frequent question topics addressed to the assistant chatbot. This result meant that the survivors wanted to know how to punish offenders, gather evidence, and list support centers. Also, $30 \%$ of the questions omitted contextual information, and $10 \%$ of the questions asked consisted of only keywords (i.e., not complete sentences). To better connect the questions to conversation flow, the questions' contextual information needed to be complemented. We presented two design implications for hybrid chatbots supporting the survivors of sexual violence based on findings from the survey. First, the conversation flow of a hybrid chatbot should be designed to help report punishing offenders. Second, the connection between the chatbot's dialog flow and a survivors' question should be designed to reflect the contextual information. The main contributions of this work are as follows: 1) we present the concept of the hybrid chatbot, combining the rule-based chatbot and ML-based chatbot, to overcome the technological limitation of chatbots, 2) we designed a userfriendly system that takes into account the various characteristics of sexual violence survivors, 3) we present practical implications for designing a hybrid chatbot for supporting survivors of sexual violence.

\section{ACKNOWLEDGMENTS}

Special thanks to Hyunjin Yoo. This research was supported by the National Research Foundation of Korea(NRF) Grant funded by the Korean National Police Agency and the Ministry of Science and ICT for Police field customized research and development project. (NRF-2018M3E2A1081492). 


\section{REFERENCES}

[1] Nova Ahmed. 2016. Discussing about sexual harassment (breaking silence): The role of technology. In Conference on Human Factors in Computing Systems Proceedings, Vol. 07-12-May-2016. https://doi.org/10.1145/2851581.2892567

[2] Nazanin Andalibi, Oliver L. Haimson, Munmun De Choudhury, and Andrea Forte. 2016. Understanding social media disclosures of sexual abuse through the lenses of support seeking and anonymity. In Conference on Human Factors in Computing Systems - Proceedings. https://doi.org/10.1145/2858036.2858096

[3] Nazanin Andalibi, Oliver L. Haimson, Munmun De Choudhury, and Andrea Forte 2018. Social support, reciprocity, and anonymity in responses to sexual abuse disclosures on social media. ACM Transactions on Computer-Human Interaction 25, 5 (2018). https://doi.org/10.1145/3234942

[4] Tobias Bauer, Emre Devrim, Misha Glazunov, William Lopez Jaramillo, Balaganesh Mohan, and Gerasimos Spanakis. 2020. \#MeTooMaastricht: Building a chatbot to assist survivors of sexual harassment. In Communications in Computer and Information Science, Vol. 1167 CCIS. https://doi.org/10.1007/978-3030-43823-4\{ $\} 41$

[5] Manon Ceelen, Tina Dorn, Flora S. van Huis, and Udo J.L. Reijnders. 2019. Characteristics and Post-Decision Attitudes of Non-Reporting Sexual Violence Victims. Fournal of Interpersonal Violence 34, 9 (2019). https://doi.org/10.1177/ 0886260516658756

[6] K Charmaz. 2014. Constructing Grounded Theory - Kathy Charmaz - Google Books. Google Scholar (2014)

[7] Munmun De Choudhury and Sushovan De. 2014. Mental health discourse on reddit: Self-disclosure, social support, and anonymity. In Proceedings of the 8th International Conference on Weblogs and Social Media, ICWSM 2014.

[8] Lei Cui, Shaohan Huang, Furu Wei, Chuanqi Tan, Chaoqun Duan, and Ming Zhou. 2017. Superagent: A customer service chatbot for E-commerce websites. In ACL 2017 - 55th Annual Meeting of the Association for Computational Linguistics, Proceedings of System Demonstrations. https://doi.org/10.18653/v1/P17-4017

[9] Jacob Devlin, Ming Wei Chang, Kenton Lee, and Kristina Toutanova. 2019. BERT: Pre-training of deep bidirectional transformers for language understanding. In NAACL HLT 2019 - 2019 Conference of the North American Chapter of the Association for Computational Linguistics: Human Language Technologies - Proceedings of the Conference, Vol. 1.

[10] Jessica Eaton. 2019. 'Logically, I know I'm not to blame but I still feel to blame' exploring and measuring victim blaming and self-blame of women who have been subjected to sexual violence. (12 2019).

[11] David Finkelhor, Kimberly J. Mitchell, and Janis Wolak. 2000. Online Victimization A Report on the Nation's Young People. Technical Report.

[12] Kathleen Kara Fitzpatrick, Alison Darcy, and Molly Vierhile. 2017. Delivering Cognitive Behavior Therapy to Young Adults With Symptoms of Depression and Anxiety Using a Fully Automated Conversational Agent (Woebot): A Randomized Controlled Trial. FMIR Mental Health 4, 2 (2017). https://doi.org/10.2196/mental. 7785

[13] Abigail Garrett and Naeemul Hassan. 2019. Understanding the silence of sexual harassment victims through the \#Whyididntreport movement. In Proceedings of the 2019 IEEE/ACM International Conference on Advances in Social Networks Analysis and Mining, ASONAM 2019. https://doi.org/10.1145/3341161.3343700
[14] Angela J. Jacques-Tiura, Rifky Tkatch, Antonia Abbey, and Rhiana Wegner. 2010. Disclosure of sexual assault: Characteristics and implications for posttraumatic sress symptoms among African American and Caucasian survivors. Fournal of Trauma and Dissociation 11, 2 (2010). https://doi.org/10.1080/15299730903502938

[15] Adam N. Joinson and Carina B. Paine. 2012. Self-disclosure, Privacy and the Internet. In Oxford Handbook of Internet Psychology. https://doi.org/10.1093/ oxfordhb/9780199561803.013.0016

[16] Aparup Khatua, Erik Cambria, and Apalak Khatua. 2018. Sounds of silence breakers: Exploring sexual violence on Twitter. In Proceedings of the 2018 IEEE/ACM International Conference on Advances in Social Networks Analysis and Mining, ASONAM 2018. https://doi.org/10.1109/ASONAM.2018.8508576

[17] Soomin Kim, Joonhwan Lee, and Gahgene Gweon. 2019. Comparing data from chatbot and web surveys effects of platform and conversational style on survey response quality. In Conference on Human Factors in Computing Systems Proceedings. https://doi.org/10.1145/3290605.3300316

[18] Yingchi Liu, Quanzhi Li, Xiaozhong Liu, Qiong Zhang, and Luo Si. 2019. Sexual harassment story classification and key information identification. In International Conference on Information and Knowledge Management, Proceedings. https://doi.org/10.1145/3357384.3358146

[19] Medical University of South Carolina. 1992. Rape in America: Report to the Nation. Technical Report. Medical University of South Carolina.

[20] Gyorgy Molnar and Zoltan Szuts. 2018. The Role of Chatbots in Formal Education. In SISY 2018 - IEEE 16th International Symposium on Intelligent Systems and Informatics, Proceedings. https://doi.org/10.1109/SISY.2018.8524609

[21] Parmy Olson. 2018. This Chatbot Is Helping People Track Harassment At Work. https://www.forbes.com/sites/parmyolson/2018/03/02/chatbot-spotsexual-harassment-ai/

[22] Matthew D. Pickard, Catherine A. Roster, and Yixing Chen. 2016. Revealing sensitive information in personal interviews: Is self-disclosure easier with humans or avatars and under what conditions? Computers in Human Behavior 65 (2016). https://doi.org/10.1016/j.chb.2016.08.004

[23] Project ReSET. [n.d.]. Project ReSET. https://twitter.com/ProjReSET

[24] Robert Ambrogi. 2016. Chatbot Helps Crime Victims Guage Their Legal Options. https://www.lawsitesblog.com/2016/12/chatbot-helps-crime-victimsguage-legal-options.html

[25] Rollo Carpenter. [n.d.]. Jabberwacky. http://jabberwacky.com/

[26] Barbara Olasov Rothbaum, Edna B. Foa, David S. Riggs, Tamera Murdock, and William Walsh. 1992. A prospective examination of post-traumatic stress disorder in rape victims. Fournal of Traumatic Stress 5, 3 (1992). https://doi.org/10.1002/ jts. 2490050309

[27] Bayan Abu Shawar and Eric Atwell. 2015. ALICE chatbot: Trials and outputs. Computacion y Sistemas 19, 4 (2015). https://doi.org/10.13053/CyS-19-4-2326

[28] Chelsea Spencer, Allen Mallory, Michelle Toews, Sandra Stith, and Leila Wood. 2017. Why Sexual Assault Survivors Do Not Report to Universities: A Feminist Analysis. Family Relations 66, 1 (2017). https://doi.org/10.1111/fare.12241

[29] Joseph Weizenbaum. 1966. ELIZA-A computer program for the study of natural language communication between man and machine. Commun. ACM 9, 1 (1966). https://doi.org/10.1145/365153.365168

[30] Wikipedia. [n.d.]. SmarterChild. https://en.wikipedia.org/wiki/SmarterChild 